\title{
INTERSECTION THEORY FOR TWISTED COHOMOLOGIES AND TWISTED RIEMANN'S PERIOD RELATIONS I
}

\author{
KOJI CHO AND KEIJI MATSUMOTO \\ To the memory of Professor Michitake Kita
}

\section{Introduction}

The beta function $B(\alpha, \beta)$ is defined by the following integral

$$
B(\alpha, \beta)=\int_{0}^{1} t^{\alpha-1}(1-t)^{\beta-1} d t
$$

where $\arg t=\arg (1-t)=0, \Re \alpha, \Re \beta>0$, and the gamma function $\Gamma(\alpha)$ by

$$
\Gamma(\alpha)=\int_{0}^{\infty} t^{\alpha-1} e^{-t} d t
$$

where $\arg t=0, \Re \alpha>0$. By the use of the well known formulae

$$
B(\alpha, \beta)=\frac{\Gamma(\alpha) \Gamma(\beta)}{\Gamma(\alpha+\beta)}, \quad \Gamma(\alpha+1)=\alpha \Gamma(\alpha), \quad \Gamma(\alpha) \Gamma(1-\alpha)=\frac{\pi}{\sin \pi \alpha},
$$

we get the following formula:

$$
B(\alpha, \beta) B(-\alpha,-\beta)=2 \pi i\left(\frac{1}{\alpha}+\frac{1}{\beta}\right)\left(-\frac{\exp (2 \pi i(\alpha+\beta))-1}{(\exp (2 \pi i \alpha)-1)(\exp (2 \pi i \beta)-1)}\right) .
$$

If we regard the interval $(0,1)$ of integration as a twisted cycle defined by the multi-valued function $t^{\alpha}(1-t)^{\beta}$, the factor

$$
-\frac{\exp (2 \pi i(\alpha+\beta))-1}{(\exp (2 \pi i \alpha)-1)(\exp (2 \pi i \beta)-1)}
$$

is nothing but the twisted self-intersection number ([KY1]) of the cycle $(0,1)$. It is quite natural to think that the factor

Received May 23, 1994. 


$$
2 \pi i\left(\frac{1}{\alpha}+\frac{1}{\beta}\right)
$$

should be the "twisted self-intersection number" of the 1-form

$$
\frac{d t}{t}+\frac{d t}{1-t}
$$

so that the above formula should be thought of a twisted version of Riemann's period relation.

This paper establishes the intersection theory for twisted cocycles and the twisted Riemann's period relation connecting the intersection theories for twisted cycles [KY1] and for twisted cocycles.

In the following we explain the results of this paper using as plain language as possible; the notion and notation used are rigorously fixed in the text. Let $x_{0}, \ldots, x_{n}$ be $n+1$ distinct points on $\boldsymbol{P}^{1}$, and

$$
\omega=\sum_{j=0}^{n} \alpha_{j} \frac{d t}{t-x_{j}}, \quad\left(\sum_{j=0}^{n} \alpha_{j}=0, \alpha_{j} \notin \mathbf{N}-\{0\}\right)
$$

a connection form. The first twisted cohomology group

$$
H^{1}(U, L) \simeq \mathbf{H}^{1}\left(\boldsymbol{P}^{1},\left(\Omega^{\cdot}(\log D), \nabla\right)\right), U:=\boldsymbol{P}^{1}-D
$$

with respect to the connection $\nabla=d+\omega \wedge$ is known to be isomorphic to

$$
\Gamma\left(\boldsymbol{P}^{1}, \Omega^{1}(\log D)\right) / \mathbf{C} \cdot \omega, \quad D:=x_{0}+\cdots+x_{n},
$$

where

$$
L:=\operatorname{ker}\left(\left.\nabla\right|_{U}:\left.\mathfrak{O}_{U} \rightarrow \Omega_{P^{1}}^{1}(\log D)\right|_{U}\right)
$$

is a local system on $U$ defined by $\nabla$.

The dual of the cohomology group $H^{1}(U, L)$ is given by the cohomology group with compact support $H_{c}^{1}\left(U, L^{\vee}\right)$, where $L^{\vee}$ is the local system defined by the connection $\nabla^{\vee}:=d-\omega \wedge$ dual to $\nabla$. We show that the dual cohomology group is isomorphic to $\Gamma\left(\boldsymbol{P}^{1}, \Omega^{1}(\log D)\right) / \mathbf{C} \cdot(-\omega)$. Since there is a natural dual pairing between the two cohomology groups $H^{1}(U, L)$ and $H_{c}^{1}\left(U, L^{\vee}\right)$, there should exist the induced bilinear form on the spaces $\Gamma\left(\boldsymbol{P}^{1}, \Omega^{1}(\log D)\right) / \mathbf{C} \cdot \omega$ and $\Gamma\left(\boldsymbol{P}^{1}, \Omega^{1}(\log D)\right) / \mathbf{C} \cdot(-\omega)$. By using elements

$$
\varphi_{j}=\frac{d t}{t-x_{j}}-\frac{d t}{t-x_{j+1}} \in \Gamma\left(\boldsymbol{P}^{1}, \Omega^{1}(\log D)\right), \quad 1 \leq j \leq n-1,
$$

we give bases for the spaces above by 
$\varphi_{j}^{+} \in \Gamma\left(\boldsymbol{P}^{1}, \Omega^{1}(\log D)\right) / \mathbf{C} \cdot \omega, \overline{\varphi_{j}^{-}} \in \Gamma\left(\boldsymbol{P}^{1}, \Omega^{1}(\log D)\right) / \mathbf{C} \cdot(-\omega), 1 \leq j \leq n-1$, where $\varphi_{j}^{+}$and $\overline{\varphi_{j}^{-}}$are the images of $\varphi_{j}$ by the natural projections from $\Gamma\left(\boldsymbol{P}^{1}\right.$, $\left.\Omega^{1}(\log D)\right)$. Our first main theorem gives explicitly the bilinear form, which turns out to be symmetric and will be called the intersection form:

$$
\begin{aligned}
\left\langle\varphi_{j}^{+}, \varphi_{j}^{-}\right\rangle & =2 \pi i\left(\frac{1}{\alpha_{j}}+\frac{1}{\alpha_{j+1}}\right), \\
\left\langle\varphi_{j}^{+}, \varphi_{j+1}^{-}\right\rangle & =\left\langle\varphi_{j+1}^{+}, \overline{\varphi_{j}^{-}}\right\rangle=-\frac{2 \pi i}{\alpha_{j+1}}, \\
\left\langle\varphi_{j}^{+}, \varphi_{k}^{-}\right\rangle & =0 \quad \text { if }|j-k| \geq 2 .
\end{aligned}
$$

Our second main theorem states the relation between the three pairings: the intersection form for twisted cohomologies, that for twisted homologies, and the pairing of twisted homologies and twisted cohomologies, i.e. integrals. Let

$$
\gamma_{j}^{+} \in H_{1}\left(U, L^{\vee}\right), \quad \delta_{j}^{-} \in H_{1}(U, L), \quad j=1, \ldots, n-1
$$

be any bases of twisted cycles (the notation is slightly different from that in [KY1]) and

$$
\begin{aligned}
\xi_{j}^{+} & \in \Gamma\left(\boldsymbol{P}^{1}, \Omega^{1}(\log D)\right) / \mathbf{C} \cdot \omega, \quad j=1, \ldots, n-1, \\
\eta_{j}^{-} & \in \Gamma\left(\boldsymbol{P}^{1}, \Omega^{1}(\log D)\right) / \mathbf{C} \cdot(-\omega), \quad j=1, \ldots, n-1,
\end{aligned}
$$

be any bases of twisted cocycles; let $I_{h}$ and $I_{c h}$ be the intersection matrices:

$$
I_{h}=\left(\begin{array}{ccc}
\left\langle\gamma_{1}^{+}, \delta_{1}^{-}\right\rangle & \cdots & \left\langle\gamma_{1}^{+}, \delta_{n-1}^{-}\right\rangle \\
\vdots & & \vdots \\
\left\langle\gamma_{n-1}^{+}, \delta_{1}^{-}\right\rangle & \cdots & \left\langle\gamma_{n-1}^{+}, \delta_{n-1}^{-}\right\rangle
\end{array}\right), \quad I_{c h}=\left(\begin{array}{ccc}
\left\langle\xi_{1}^{+}, \eta_{1}^{-}\right\rangle & \cdots & \left\langle\xi_{1}^{+}, \eta_{n-1}^{-}\right\rangle \\
\vdots & & \vdots \\
\left\langle\xi_{n-1}^{+}, \eta_{1}^{-}\right\rangle & \cdots & \left\langle\xi_{n-1}^{+}, \eta_{n-1}^{-}\right\rangle
\end{array}\right) .
$$

The intersection matrix $I_{h}$ can be explicitly computed [KY1]; take for instance bases $\gamma_{j}^{+}$and $\delta_{j}^{-}:=\varphi_{j}^{-}$as follows: let us assume for simplicity that the $x_{j}$ 's are all real and are arranged as $x_{0}<x_{1}<\cdots<x_{n}$, and $u_{0}$ a branch of the multi-valued function $u=\Pi\left(t-x_{j}\right)^{\alpha_{j}}$ defined on the lower half $t$-plane. We define special cycles by

$$
\begin{aligned}
& r_{j}^{+}=\left(p_{j}, \vec{q}_{j+1}\right) \otimes u_{0}+\frac{1}{c_{j}-1} S_{j} \otimes u_{0}-\frac{1}{c_{j+1}-1} S_{j+1} \otimes u_{0}, \\
& r_{j}^{-}=\left(p_{j}, \vec{q}_{j+1}\right) \otimes u_{0}^{-1}-\frac{c_{j}}{c_{j}-1} S_{j} \otimes u_{0}^{-1}+\frac{c_{j+1}}{c_{j+1}-1} S_{j+1} \otimes u_{0}^{-1}, c_{j}=\exp 2 \pi i \alpha_{j},
\end{aligned}
$$

where $S_{k}$ is a positively oriented circle with center $x_{k}$ and with starting point $p_{k}$ 
or $q_{k}$; see Figure.

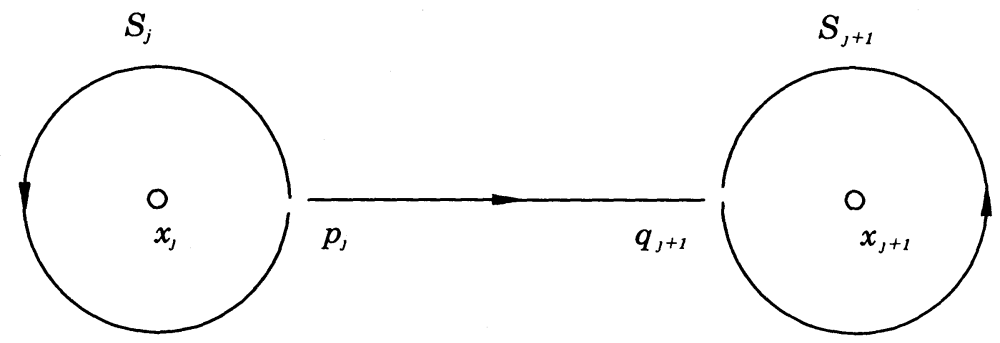

Figure

Then the intersection matrix for these special bases turns out to be

$$
I_{h}=\left(\begin{array}{cccccc}
-d_{12} / d_{1} d_{2} & 1 / d_{2} & 0 & \cdots & 0 & 0 \\
c_{2} / d_{2} & -d_{23} / d_{2} d_{3} & \cdots & & 0 & 0 \\
0 & \vdots & & & \vdots & \vdots \\
\vdots & & & & 0 \\
0 & 0 & & \cdots & -d_{n-2, n-1} / d_{n-2} d_{n-1} & 1 / d_{n-1} \\
0 & 0 & \cdots & 0 & c_{n-1} / d_{n-1} & -d_{n-1, n} / d_{n-1} d_{n}
\end{array}\right) \text {, }
$$

where $d_{j}=c_{j}-1, d_{j k}=c_{j} c_{k}-1$. It is easy to see that

$$
{ }^{t} I_{h}^{-1}=\frac{-1}{d_{1 \cdots n}}\left(\begin{array}{ccccc}
d_{1} d_{2 \cdots n} & d_{1} c_{2} d_{3 \cdots n} & d_{1} c_{23} d_{4 \cdots n} & \cdots & d_{1} c_{2 \cdots n-1} d_{n} \\
d_{1} d_{3 \cdots n} & d_{12} d_{3 \cdots n} & d_{12} c_{3} d_{4 \cdots n} & \cdots & d_{12} c_{3 \cdots n-1} d_{n} \\
d_{1} d_{4 \cdots n} & d_{12} d_{4 \cdots n} & d_{123} d_{4 \cdots n} & \cdots & d_{123} c_{4 \cdots n-1} d_{n} \\
\vdots & \vdots & \vdots & & \vdots \\
d_{1} d_{n} & d_{12} d_{n} & d_{123} d_{n} & \cdots & d_{1 \cdots n-1} d_{n}
\end{array}\right),
$$

where $c_{j k \ldots}=c_{j} c_{k} \cdots, d_{j k \cdots}=c_{j} c_{k} \cdots-1$. Let us arrange the integrals (periods) as follows:

$$
P^{+}=\left(\begin{array}{ccc}
\int_{\gamma_{1}^{+}} \xi_{1}^{+} & \cdots & \int_{\gamma_{n-1}^{+}} \xi_{1}^{+} \\
\vdots & & \vdots \\
\int_{\gamma_{1}^{+}} \xi_{n-1}^{+} & \cdots & \int_{\gamma_{n-1}^{+}} \xi_{n-1}^{+}
\end{array}\right), \quad P^{-}=\left(\begin{array}{ccc}
\int_{\delta_{1}^{-}} \eta_{1}^{-} & \cdots & \int_{\delta_{n-1}^{-}} \eta_{1}^{-} \\
\vdots & & \vdots \\
\int_{\delta_{1}^{-}} \eta_{n-1}^{-} & \cdots & \int_{\delta_{n-1}^{-1}} \eta_{n-1}^{-}
\end{array}\right) .
$$

Here the integral $\int_{\gamma_{1}^{+}} \xi^{+}$(resp. $\int_{\delta^{-}} \eta^{-}$) of a twisted cocycle $\xi^{+}$(resp. $\eta^{-}$) over a twisted cycle $\gamma^{+} \in H_{1}\left(U, L^{\vee}\right)$ (resp. $\delta^{-} \in H_{1}(U, L)$ ) is defined as follows: for a 
twisted cocycle $\xi^{+}$(resp. $\eta^{-}$) take a representing form $\xi$ (resp. $\eta$ ) of $\Gamma\left(\boldsymbol{P}^{1}\right.$, $\left.\Omega^{1}(\log D)\right)$ and for a twisted cycle $\gamma^{+}\left(\right.$resp. $\left.\delta^{-}\right)$take a representing twisted chain $\sum_{i} g_{i} \otimes u_{i}$ (resp. $\sum_{i} g_{i}^{\prime} \otimes u_{i}^{-1}$ ), where $g_{i}$ (resp. $g_{i}^{\prime}$ ) is a topological chain and $u_{i}$ (resp. $u^{-1}$ ) is a branch of the multi-valued function

$$
u=\prod_{j=0}^{n}\left(t-x_{j}\right)^{\alpha_{j}} \quad\left(\text { resp. } u^{-1}\right)
$$

along $g_{i}\left(\right.$ resp. $\left.g_{i}^{\prime}\right)$; then

$$
\int_{\gamma^{+}} \xi^{+}:=\sum_{i} \int_{g_{i}} u_{i} \xi, \quad \int_{\delta^{-}} \eta^{-}:=\sum_{i} \int_{g_{i}^{\prime}} u_{i}^{-1} \eta
$$

which are independent of the choice of representatives. Our theorem reads

$$
P^{+t} I_{h}^{-1 t} P^{-}=I_{c h} \text {, i.e. }{ }^{t} P^{-} I_{c h}^{-1} P^{+}={ }^{t} I_{h} .
$$

We would like to call these identities twisted Riemann's period relations because it resembles Riemann's period relation for a basis of holomorphic 1 -forms $\omega_{1}, \ldots$, $\omega_{g}$ and a $\boldsymbol{Z}$-basis of cycles $\gamma_{1}, \ldots, \gamma_{2 g}$ on a compact Riemann surface of genus $g$. The period matrix $P$ and the intersection matrix $I_{h}$ of cycles are

$$
P=\left(\begin{array}{ccc}
\int_{\gamma_{1}} \omega_{1} & \cdots & \int_{\gamma_{2 g}} \omega_{1} \\
\vdots & & \vdots \\
\int_{\gamma_{1}} \omega_{g} & \cdots & \int_{\gamma_{2 g}} \omega_{g}
\end{array}\right), \quad I_{h}=\left(\begin{array}{ccc}
\left\langle\gamma_{1}, \gamma_{1}\right\rangle & \cdots & \left\langle\gamma_{1}, \gamma_{2 g}\right\rangle \\
\vdots & & \vdots \\
\left\langle\gamma_{2 g}, \gamma_{1}\right\rangle & \cdots & \left\langle\gamma_{2 g}, \gamma_{2 g}\right\rangle
\end{array}\right)
$$

then Riemann's period relations are given as follows:

$$
\left(\frac{P}{P}\right){ }^{t} I_{h}^{-1}\left({ }^{t} P^{t} \bar{P}\right)=\left(\begin{array}{l}
\int \omega_{j} \wedge \omega_{k} \int \omega_{j} \wedge \bar{\omega}_{k} \\
\int \bar{\omega}_{j} \wedge \omega_{k} \int \bar{\omega}_{j} \wedge \bar{\omega}_{k}
\end{array}\right)=i\left(\begin{array}{cc}
0 & H \\
-\bar{H} & 0
\end{array}\right),
$$

where $H$ is positive definite. We remarked it not only because of the resemblance but also because we shall in [Chol] establish a theory including both Riemann's period relations.

The simplest case, i.e. $n=2$ is nothing but the formulae for $B(\alpha, \beta) B(-\alpha$, $-\beta$ ) given in the beginning; the next simplest case, i.e. $n=3$ yields ( $\$ 4$ Example 1) the famous formula

$$
\begin{aligned}
F(\alpha, \beta, \gamma ; x) F & (1-\alpha, 1-\beta, 2-\gamma ; x) \\
& =F(\alpha+1-\gamma, \beta+1-\gamma, 2-\gamma ; x) F(\gamma-\alpha, \gamma-\beta, \gamma ; x),
\end{aligned}
$$


where

$$
F(\alpha, \beta, \gamma ; x):=\sum_{n=0}^{\infty} \frac{(\alpha)_{n}(\beta)_{n}}{(\gamma)_{n}(1)_{n}} x^{n} \quad(\alpha)_{n}:=\alpha(\alpha+1) \cdots(\alpha+n-1)
$$

We cordially thank Professors K. Mimachi and M. Yoshida for their constant encouragement and stimulating discussions.

\section{§1. Preliminaries}

In the following, notation is so chosen that generalizations to Riemann surfaces of higher genus [Cho1] and to varieties of higher dimension [Cho2] would be smooth. Let $x_{0}, \ldots, x_{n}$ be $n+1$ distinct points on $\boldsymbol{P}^{1}$; put

$$
D:=x_{0}+\cdots+x_{n}, \quad U:=\boldsymbol{P}^{1}-D, \quad j: U \hookrightarrow \boldsymbol{P}^{1} .
$$

Let $\omega$ be a logarithmic 1 -form on $\boldsymbol{P}^{1}$ with poles at $D$ with residue $\alpha_{j}$ at $x_{j}$; note that

$$
\sum_{j=0}^{n} \alpha_{j}=0
$$

Consider the connection $\nabla$ with connection form $\omega$ :

$$
\nabla=d+\omega \wedge: \mathscr{O}_{\boldsymbol{P}^{1}} \rightarrow \Omega_{\boldsymbol{P}^{1}}^{1}(\log D),
$$

where $\mathscr{O}_{\boldsymbol{P}^{1}}$ is the sheaf of holomorphic functions on $\boldsymbol{P}^{1}, \Omega_{\boldsymbol{P}^{1}}^{1}$ the sheaf of holomorphic 1 -forms on $\boldsymbol{P}^{1}$ and $\Omega_{\boldsymbol{P}^{1}}^{1}(\log D)$ the sheaf of meromorphic 1 -forms with logarithmic singularities only on $D$. Let $L$ be a local system on $U$ defined by

$$
L:=\operatorname{ker}\left(\left.\nabla\right|_{U}:\left.\mathscr{O}_{U} \rightarrow \Omega_{P^{1}}^{1}(\log D)\right|_{U}\right),
$$

where $\mathscr{O}_{U}$ is the sheaf of holomorphic functions on $U$.

We are going to present several isomorphisms for two hypercohomologies; they shall be made explicit in the next section; the definition of hypercohomology shall be also given in $\S 2.2$. If $\alpha_{j} \notin \mathbf{N}-\{0\}$ then the following quasi-isomorphism [Del1] holds

$$
\begin{aligned}
\boldsymbol{R} j_{*} L \underset{\text { qis }}{\simeq} & \left(\Omega^{*}(\log D), \nabla\right) \\
& :=\cdots 0 \rightarrow \mathscr{O}_{\boldsymbol{P}^{1}} \stackrel{\nabla}{\rightarrow} \Omega_{\boldsymbol{P}^{1}}^{1}(\log D) \rightarrow 0 \cdots,
\end{aligned}
$$

which leads to 


$$
\begin{aligned}
H^{1}(U, L) & \simeq \mathbf{H}^{1}\left(\boldsymbol{P}^{1},\left(\Omega^{\bullet}(\log D), \nabla\right)\right) \\
& \simeq \Gamma\left(\boldsymbol{P}^{1}, \Omega^{1}(\log D)\right) / \mathbf{C} \cdot \omega,
\end{aligned}
$$

where the last isomorphism is derived by the (Hodge-to-logarithmic de Rham) spectral sequence:

$$
E_{1}^{p q} \simeq H^{q}\left(\boldsymbol{P}^{1}, \Omega^{p}(\log D)\right) \Rightarrow \mathbf{H}^{p+q}\left(\boldsymbol{P}^{1},\left(\Omega^{\bullet}(\log D), \nabla\right)\right),
$$

and $E_{1}^{p q}=0$ if $q>0$.

On the other hand by the Poincaré-Verdier duality [EV1], (i.e. by performing $\left.\boldsymbol{R} \mathscr{H} \operatorname{om}\left(\cdot, \mathbf{C}_{\boldsymbol{P}^{1}}\right)\right)$ we have:

$$
\begin{aligned}
& j_{!} L^{\vee} \underset{\text { qis }}{\simeq}\left(\Omega^{\cdot}(\log D)(-D), \nabla^{\vee}\right) \\
& :=\cdots 0 \rightarrow \mathscr{O}_{\boldsymbol{P}^{1}}(-D) \stackrel{\nabla^{\vee}}{\rightarrow} \Omega_{\boldsymbol{P}^{1}}^{1}(\log D)(-D) \simeq \Omega_{\boldsymbol{P}^{1}}^{1} \rightarrow 0 \cdots,
\end{aligned}
$$

where $\nabla^{\vee}:=d-\omega$, and ! means the zero-extension; this leads to

$$
\begin{aligned}
H_{c}^{1}\left(U, L^{\vee}\right) & \simeq \mathbf{H}^{1}\left(\boldsymbol{P}^{1},\left(\Omega^{\vee}(\log D)(-D), \nabla^{\vee}\right)\right) \\
& \simeq \operatorname{ker}\left(\nabla^{\vee}: H^{1}\left(\boldsymbol{P}^{1}, \mathcal{O}_{\boldsymbol{P}^{1}}(-D)\right) \rightarrow H^{1}\left(P^{1}, \Omega_{\boldsymbol{P}^{1}}^{1}\right)\right) \\
& =\operatorname{ker}\left(-\omega: H^{1}\left(\boldsymbol{P}^{1}, \widehat{O}_{\boldsymbol{P}^{1}}(-D)\right) \rightarrow H^{1}\left(P^{1}, \Omega_{\boldsymbol{P}^{1}}^{1}\right)\right),
\end{aligned}
$$

where $H_{c}$ means cohomology with compact support, and the second isomorphism is derived by the spectral sequence:

$$
E_{1}^{p q}=H^{q}\left(\boldsymbol{P}^{1}, \Omega^{p}(\log D)(-D)\right) \Rightarrow \mathbf{H}^{p+q}\left(\boldsymbol{P}^{1},\left(\Omega^{\cdot}(\log D)(-D), \nabla^{\vee}\right)\right),
$$

and $E_{1}^{p q}=0$ if $q=0$. Notice that the duality between $\left(\Omega^{\circ}(\log D), \nabla\right)$ and $\left(\Omega^{\circ}(\log D)(-D), \nabla^{\vee}\right)$ holds without any condition for $\alpha_{j}[$ EV2]. Notice also that the duality above between $\Gamma\left(\boldsymbol{P}^{1}, \Omega^{1}(\log D) / \mathbf{C} \cdot \omega\right.$ and $\operatorname{ker}\left(-\omega: H^{1}\left(\boldsymbol{P}^{1}, \mathscr{O}_{\boldsymbol{P}^{1}}(-D)\right)\right.$ $\left.\rightarrow H^{1}\left(\boldsymbol{P}^{1}, \Omega_{\boldsymbol{P}^{1}}^{1}\right)\right)$ is induced by the Serre duality. We denote by $\varphi^{+}\left(\right.$resp. $\left.\varphi^{-}\right)$the im age of $\varphi \in \Gamma\left(\boldsymbol{P}^{1}, \Omega^{1}(\log D)\right)$ under the natural projection to $\Gamma\left(\boldsymbol{P}^{1}, \Omega^{1}(\log D) / \mathbf{C} \cdot \omega\right.$ (resp. $\Gamma\left(\boldsymbol{P}^{1}, \Omega^{1}(\log D) / \mathbf{C} \cdot(-\omega)\right)$.

\section{§2. Intersection theory for twisted cocycles}

Consider the following exact sequence of complexes, which will be referred to as the basic sequence:

$$
0 \rightarrow\left(\Omega^{\bullet}(\log D)(-D), \nabla^{\vee}\right) \stackrel{\iota}{\rightarrow}\left(\Omega^{\cdot}(\log D), \nabla^{\vee}\right) \rightarrow\left(\bigoplus_{j=0}^{n} \mathbf{C}_{x_{j}} \stackrel{\times \text { res }}{\rightarrow} \bigoplus_{j=0}^{n} \mathbf{C}_{x_{j}}\right) \rightarrow 0 ;
$$

that is 


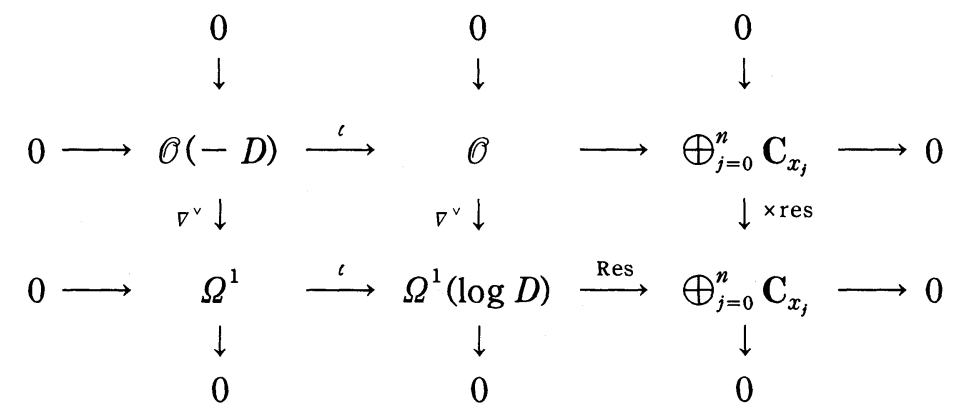

where

$$
\times \text { res }:\left(c_{0}, \ldots, c_{n}\right) \rightarrow\left(-\alpha_{0} c_{0}, \ldots,-\alpha_{n} c_{n}\right) .
$$

If $\alpha_{j} \neq 0$ then $\times$ res is isomorphic, so we have the following isomorphism

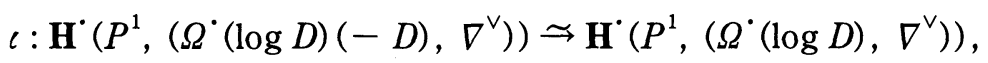

in particular,

$$
\iota: \operatorname{ker}\left(-\omega: H^{1}\left(\boldsymbol{P}^{1}, \mathcal{O}_{\boldsymbol{P}^{1}}(-D)\right) \rightarrow H^{1}\left(\boldsymbol{P}^{1}, \Omega_{\boldsymbol{P}^{1}}^{1}\right)\right) \widetilde{\hookrightarrow} \Gamma\left(\boldsymbol{P}^{1}, \Omega^{1}(\log D)\right) / \mathbf{C} \cdot(-\omega) .
$$

We shall explicitly give the inverse of the isomorphism $\iota$. We first define a homomorphism: $\tau: \Gamma\left(\Omega^{1}(\log D)\right) / \mathbf{C} \cdot(-\omega) \rightarrow \operatorname{ker}\left(-\omega: H^{1}(\mathscr{O}(-D)) \rightarrow H^{1}\left(\Omega^{1}\right)\right)$ and secondly prove that this gives the inverse of the natural isomorphism $\iota$.

\section{\$2.1. Definition of $\tau$}

The corresponding long exact sequences of (1) read

$$
\begin{array}{cccc}
\longrightarrow & H^{0}(\mathbb{O}) \longrightarrow \bigoplus_{j=0}^{n} \mathbf{C}_{x_{j}} \stackrel{\delta}{ } \longrightarrow H^{1}(\mathscr{O}(-D)) \\
& & & \\
\longrightarrow & H^{0}\left(\Omega^{1}(\log D)\right) \stackrel{\text { Res }}{\longrightarrow} \bigoplus_{j=0}^{n} \mathbf{C}_{x_{j}} \longrightarrow H^{1}\left(\Omega^{1}\right)
\end{array}
$$

where $\delta$ is the connecting homomorphism. Tracing the above commutative diagram, we have

$$
\delta \circ(\times \text { res })^{-1} \cdot \operatorname{Res}: H^{0}\left(\Omega^{1}(\log D)\right) \rightarrow H^{1}(\mathscr{O}(-D)) ;
$$

it is immediate that this induces the isomorphism

$$
\tau: \Gamma\left(\Omega^{1}(\log D)\right) / \mathbf{C} \cdot(-\omega) \rightarrow \operatorname{ker}\left(-\omega: H^{1}(\mathscr{O}(-D)) \rightarrow H^{1}\left(\Omega^{1}\right)\right) .
$$




\section{§2.2. Naturality of $\tau$}

LEMma. $\tau=\iota^{-1}$.

Proof. Let us honestly see the homomorphism c, i.e. following the definition of hypercohomologies. A fine resolution of the complex $\left(\Omega^{*}(\log D)(-D), \nabla^{\vee}\right)$ is given by

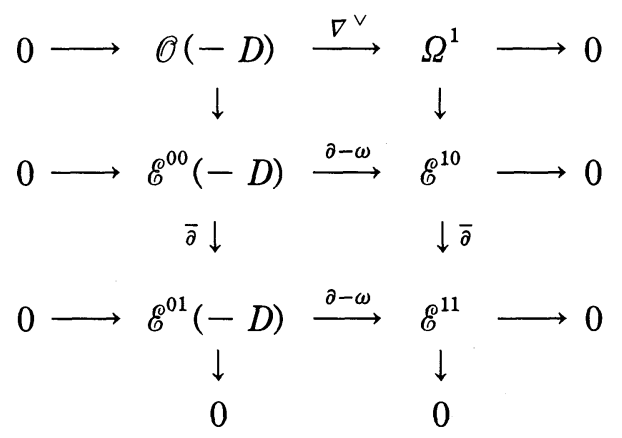

where $\mathscr{E}^{p q}$ stands for the sheaf of smooth $(p, q)$-forms on $\boldsymbol{P}^{1}$ and $\mathscr{E}^{p q}(-D)$ the sheaf of $(p, q)$-forms $g$ on $\boldsymbol{P}^{1}$ such that $g / t_{j}$ is smooth for a local parameter $t_{j}$ around $x_{j}$. The associated single complex is

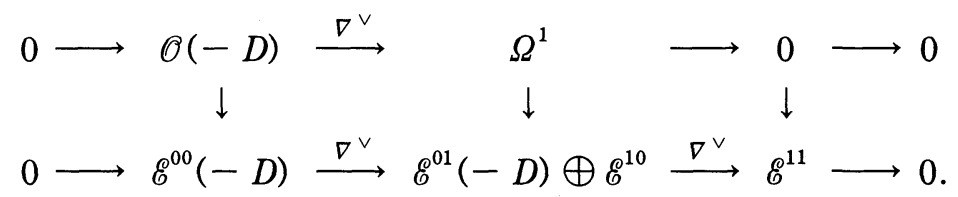

Thus we have

$$
\mathbf{H}^{1}\left(\boldsymbol{P}^{1},\left(\Omega^{\cdot}(\log D)(-D), \nabla^{\vee}\right)\right) \simeq \frac{\operatorname{ker}\left\{\Gamma\left(\mathscr{E}^{01}(-D)\right) \oplus \Gamma\left(\mathscr{E}^{10}\right) \rightarrow \Gamma\left(\mathscr{E}^{11}\right)\right\}}{\nabla^{\vee} \Gamma\left(\mathscr{E}^{00}(-D)\right)} ;
$$

for $\eta \in \Gamma\left(\boldsymbol{P}^{1}, \Omega^{1}(\log D)\right)$, we denote by $\eta^{\vee}$ the image of $\eta^{-} \in \Gamma\left(\Omega^{1}(\log D)\right) / \mathbf{C}$ $\cdot(-\omega)$ under $\tau$. Since the Dolbeault resolution implies

$$
\left.H^{1}\left(\boldsymbol{P}^{1}, \mathscr{O}_{\boldsymbol{P}^{1}}(-D)\right) \simeq \frac{\Gamma\left(\mathscr{E}^{01}(-D)\right)}{\bar{\partial} \Gamma\left(\mathscr{E}^{00}(-D)\right)}, \quad H^{1}\left(\boldsymbol{P}^{1}, \Omega_{\boldsymbol{P}^{1}}^{1}\right)\right) \simeq \frac{\Gamma\left(\mathscr{E}^{11}\right)}{\bar{\partial} \Gamma\left(\mathscr{E}^{10}\right)},
$$

$\nabla^{\vee}=d-\omega$ annihilates $\eta^{\vee}$ means that there exists $\mu \in \Gamma\left(\mathscr{E}^{10}\right)$ such that

$$
(d-\omega) \eta^{\vee}=\bar{\partial} \mu,
$$

namely,

$$
\nabla^{\vee}\left(\eta^{\vee}+\mu\right)=0
$$


This gives an explicit expression of the isomorphism

$$
\begin{aligned}
\operatorname{ker}\left(\nabla^{\vee}: H^{1}(\mathscr{O}(-D)) \rightarrow H^{1}\left(\Omega^{1}\right)\right) & \stackrel{\simeq}{\rightarrow} \mathbf{H}^{1}\left(\left(\Omega^{\bullet}(\log D)(-D), \nabla^{\vee}\right)\right) \\
& \simeq \frac{\operatorname{ker}\left\{\Gamma\left(\mathscr{E}^{01}(-D)\right) \oplus \Gamma\left(\mathscr{E}^{10}\right) \rightarrow \Gamma\left(\mathscr{E}^{11}\right)\right\}}{\nabla^{\vee} \Gamma\left(\mathscr{E}^{00}(-D)\right)} .
\end{aligned}
$$

Similarly a single fine resolution of $\left(\Omega^{*}(\log D), \nabla^{\vee}\right)$ :

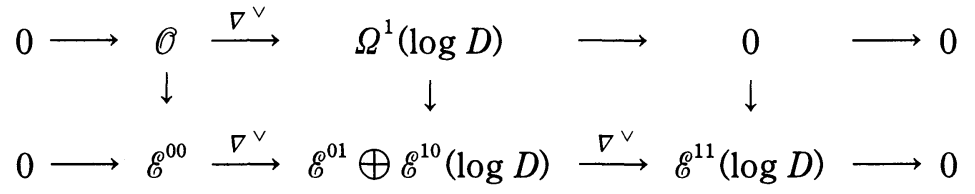

gives

$$
\mathbf{H}^{1}\left(\boldsymbol{P}^{1},\left(\Omega^{\cdot}(\log D), \nabla^{\vee}\right)\right) \simeq \frac{\operatorname{ker}\left\{\Gamma\left(\mathscr{E}^{01}\right) \oplus \Gamma\left(\mathscr{E}^{10}(\log D)\right) \rightarrow \Gamma\left(\mathscr{E}^{11}(\log D)\right)\right\}}{\nabla^{\vee} \Gamma\left(\mathscr{E}^{00}\right)} .
$$

An explicit expression of the isomorphism

$$
\begin{aligned}
\Gamma\left(\Omega^{1}(\log D)\right) / \mathbf{C} \cdot(-\omega) & \simeq \mathbf{H}^{1}\left(\boldsymbol{P}^{1},\left(\Omega^{\bullet}(\log D), \nabla^{\vee}\right)\right) \\
& \simeq \frac{\operatorname{ker}\left\{\Gamma\left(\mathscr{E}^{01}\right) \oplus \Gamma\left(\mathscr{E}^{10}(\log D)\right) \rightarrow \Gamma\left(\mathscr{E}^{11}(\log D)\right)\right\}}{\nabla^{\vee} \Gamma\left(\mathscr{E}^{00}\right)}
\end{aligned}
$$

is given by

$$
\eta^{-} \mapsto 0 \oplus \eta
$$

Summing up, a fine resolution of the basic sequence is given as follows (pay attention that rows and columns are reversed):

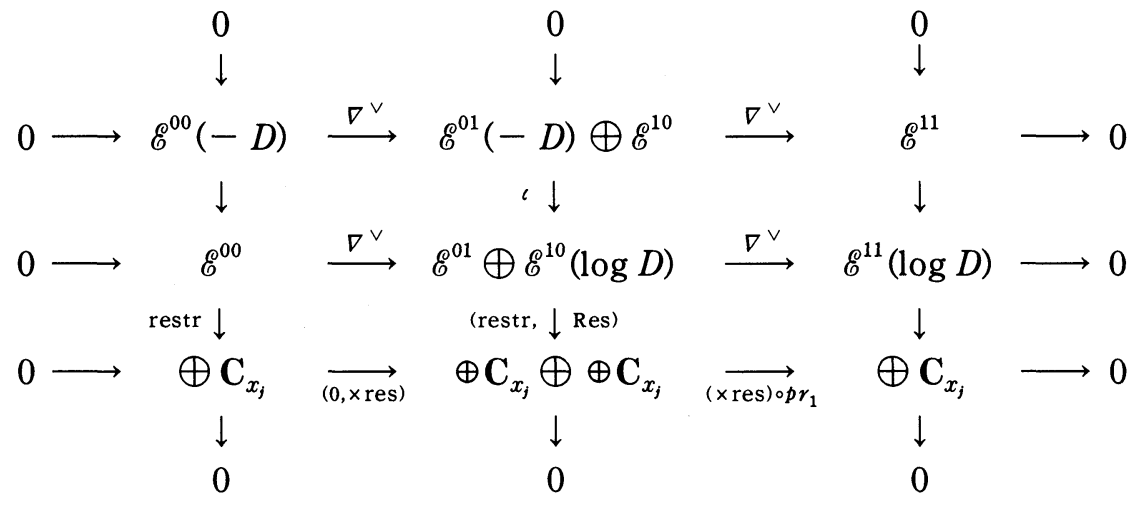

Now we are going to trace back c. Let us give $\eta \in \Gamma\left(\Omega^{1}(\log D)\right)$. We change the represetative $\eta$ to 


$$
\eta^{\prime}=\eta+\nabla^{\vee} h
$$

so that (restr, Res) $\eta^{\prime}=0$; this can be achieved by taking $h \in \Gamma\left(\mathscr{E}^{00}\right)$ so that

$$
(0, \times \text { res }) \circ \text { restr } h=(\text { restr, Res }) \eta .
$$

Then there is a form $\tilde{\eta}+\mu \in \Gamma\left(\mathscr{E}^{01}(-D) \oplus \mathscr{E}^{10}\right)$ which maps under $\iota$ to $\eta^{\prime}$; it can be readily checked that $\tilde{\eta}+\mu$ represents an element of

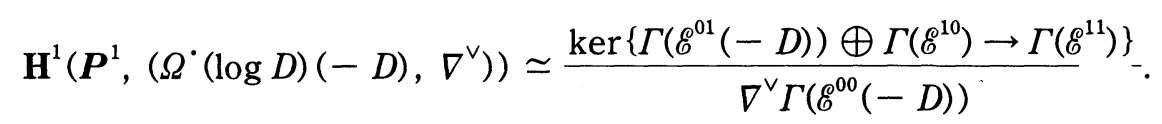

Recall the connecting homomorphism $\delta: \oplus \mathbf{C}_{x_{j}} \rightarrow H^{1}(\mathscr{O}(-D))$ used when defining $\tau$; it is exactly the same as tracing part of the above diagram:

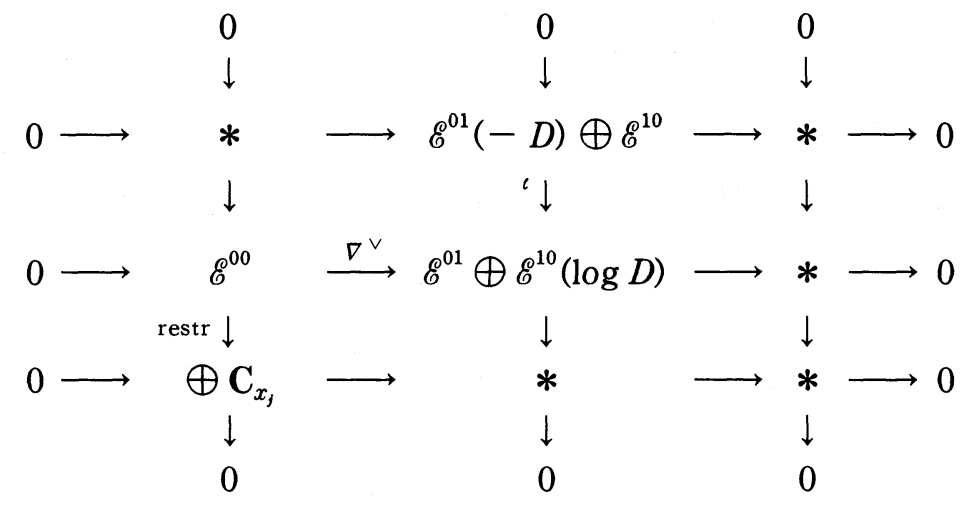

Therefore we proved that in cohomology level

$$
\tilde{\eta}=\eta^{\vee} \text { in } H^{1}(\mathscr{O}(-D)) \text {; }
$$

and so (it will be the key in $\S 3$ ),

$$
\iota\left(\eta^{\vee}+\mu\right)=\eta+\nabla^{\vee} h, \quad \mu \in \Gamma\left(\mathscr{E}^{10}\right), \quad h \in \Gamma\left(\mathscr{E}^{00}\right) .
$$

\section{\$2.3. Intersection form for cocycles}

We assume $\alpha_{j} \neq 0$. Let us fix an isomorphism

$$
\int: H^{1}\left(\Omega^{1}\right) \rightarrow \mathbf{C}
$$

by

$$
H^{1}\left(\Omega^{1}\right) \simeq H_{\mathrm{Dol}}^{1}\left(\Omega^{1}\right):=\Gamma\left(\mathscr{E}^{11}\right) / \bar{\partial} \Gamma\left(\mathscr{E}^{10}\right) \ni \zeta \mapsto \int_{\boldsymbol{P}^{1}} \zeta \in \mathbf{C} .
$$


For cocycles $\xi^{+}$and $\eta^{-}$represented by $\xi, \eta \in \Gamma\left(\Omega^{1}(\log D)\right)$, we now define the intersection form by the natural bilinear form $\langle *, *\rangle$ :

$$
\begin{gathered}
\Gamma\left(\Omega^{1}(\log D)\right) / \mathbf{C} \cdot \omega \times \Gamma\left(\Omega^{1}(\log D)\right) / \mathbf{C} \cdot(-\omega) \rightarrow \Gamma\left(\Omega^{1}(\log D)\right) / \mathbf{C} \cdot \omega \times H_{\mathrm{Dol}}^{1}(\mathscr{O}(-D)) \\
\stackrel{\text { Serre duality }}{\rightarrow} H_{\mathrm{Dol}}^{1}\left(\Omega^{1}\right) \stackrel{s}{\rightarrow} \mathbf{C} \\
\left(\xi^{+}, \eta^{-}\right) \mapsto\left(\xi^{+}, \eta^{\vee}\right) \mapsto \eta^{\vee} \wedge \xi \mapsto \int \eta^{\vee} \wedge \xi .
\end{gathered}
$$

Since $\eta^{\vee} \in \operatorname{ker}\left(-\omega: H_{\mathrm{Dol}}^{1}(\mathscr{O}(-D)) \rightarrow H_{\mathrm{Dol}}^{1}\left(\Omega^{1}\right)\right)$ and $\omega^{\vee} \sim 0$, it is well defined, and is non-degenerate thanks to non-degeneracy of the Serre duality. We compute the intersection numbers for the following forms:

$$
\omega_{i j}=\left(\frac{1}{t-x_{i}}-\frac{1}{t-x_{j}}\right) d t \in \Gamma\left(\Omega^{1}(\log D)\right), 0 \leq i \neq j \leq n
$$

Let us first explicitly write the image $\omega_{i j}^{\vee} \in H^{1}(\mathscr{O}(-D))$ under $\tau$ of $\omega_{i j}^{-}$in terms of the Čech cohomology $\stackrel{\vee}{H}^{1}(\mathcal{U}, \mathscr{O}(-D))$ with respect to the covering $\mathcal{U}=\left\{U_{j}\right\}$

$$
U_{j}:=U \cup\left\{x_{j}\right\}, j=0, \ldots, n .
$$

Claim. Let $\left(\omega_{i j}^{\vee}\right)_{\text {Cech }}$ be the expression of $\omega_{i j}^{\vee}$ in the $\check{C}$ ech cohomology, then we have

$$
\left(\omega_{i j}^{\vee}\right)_{\operatorname{cech}}=\left\{\begin{array}{lll}
1 / \alpha_{i}+1 / \alpha_{j} & \text { on } & U_{i j} \\
1 / \alpha_{i} & \text { on } & U_{i k}(k \neq i, j) \\
-1 / \alpha_{j} & \text { on } U_{j k}(k \neq i, j) \\
0 & \text { on } U_{k l}(k, l \neq i, j)
\end{array}\right.
$$

where $U_{i j}:=U_{i} \cap U_{j}$.

Here we use the convention $s_{i j}=-s_{j i}$ for $\left\{s_{i j}\right\} \in C^{1}(\mathscr{O}(-D))$, where $s_{i j} \in$ $\Gamma\left(U_{i j}, O(-D)\right), s_{j i} \in \Gamma\left(U_{j i}, O(-D)\right)$.

Proof. It is easy to see that

$$
\begin{aligned}
\omega_{i j} & \stackrel{\text { Res }}{\mapsto}\left(1 \in \mathbf{C}_{x_{i}},-1 \in \mathbf{C}_{x_{j}}, 0 \in \mathbf{C}_{x_{k}} k \neq i, j\right) \\
& \stackrel{\times \text { Res }}{\mapsto}\left(-1 / \alpha_{i} \in \mathbf{C}_{x_{i}}, 1 / \alpha_{j} \in \mathbf{C}_{x_{j}}, 0 \in \mathbf{C}_{x_{k}}\right) .
\end{aligned}
$$

The connecting map $\delta$ is given by tracing the following commutative diagram from the right-top to the left-bottom: 


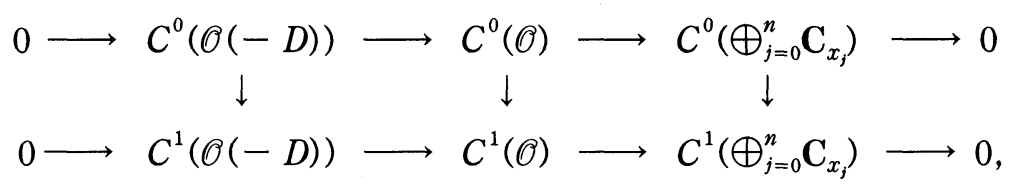

where $C^{\cdot}$ denotes a space of cochains. Thus the claim follows.

THEOREM 1. The intersection numbers for the twisted forms are

$$
\left\langle\omega_{p q}^{+}, \omega_{i j}^{-}\right\rangle=2 \pi i\left(\frac{1}{\alpha_{i}}\left(\delta_{i p}-\delta_{i q}\right)-\frac{1}{\alpha_{j}}\left(\delta_{j p}-\delta_{j q}\right)\right),
$$

where $\delta_{i p}$ is the Kronecker delta. As a result, the intersection form is symmetric.

Proof. In terms of the Čech cohomology the isomorphism $\int: H^{1}\left(\Omega^{1}\right) \stackrel{\sim}{\rightarrow} \mathbf{C}$ is given as follows: for

$$
(\zeta)_{\mathrm{cech}}=\left(\zeta_{p q}\right) \in \Omega^{1}\left(U_{p q}\right) \in \check{H}^{1}\left(U, \Omega^{1}\right), \quad \zeta \in H_{\mathrm{Dol}}^{1}\left(\Omega^{1}\right),
$$

find meromorphic 1 -forms $\eta_{p}$ on $U_{p}$ such that

$$
\eta_{q}-\eta_{p}=\zeta_{p q} \text { on } U_{p q}
$$

$\left(\left\{\eta_{p}\right\}\right.$ is called a Mittag-Leffler distribution for $\left.(\zeta)_{\text {cech }}\right)$, then [For] implies

$$
\int \zeta=2 \pi i \sum_{x \in \boldsymbol{P}^{1}} \operatorname{Res}_{x}\left\{\eta_{p}\right\}
$$

Since

$$
\left(\omega_{i j}^{\vee}\right)_{\text {cech }} \in \check{H}^{1}(\mathscr{O}(-D)), \omega_{p q} \in \Gamma\left(\Omega^{1}(\log D)\right)
$$

and $U_{a} \cap U_{b}=U(a \neq b)$, we have

$$
\left(\omega_{i j}^{\vee}\right)_{\operatorname{cech}} \cdot \omega_{p q} \in \check{H}^{1}\left(\mathcal{U}, \Omega^{1}\right) .
$$

Notice that

$$
H_{\mathrm{Dol}}^{1}\left(\Omega^{1}\right) \ni \omega_{i j}^{\vee} \wedge \omega_{p q} \leftrightarrow-\omega_{i j}^{\vee} \cdot \omega_{p q} \in \check{H}^{1}\left(\mathcal{U}, \Omega^{1}\right) .
$$

If we define $\xi=\left\{\xi_{l}\right\}$ by

$$
\begin{array}{ll}
\xi_{i}:=\omega_{p q} / \alpha_{i} & \text { a meromorphic } 1 \text {-form on } U_{i} \\
\xi_{j}:=-\omega_{p q} / \alpha_{j} & \text { a meromorphic } 1 \text {-form on } U_{j} \\
\xi_{k}:=0 & \text { on } U_{k} \quad \text { if } k \neq i, j
\end{array}
$$


it forms a Mittag-Leffler distribution for $-\left(\omega_{i j}^{\vee}\right)_{\text {cech }} \cdot \omega_{p q}$. Hence using the formula (3), we get

$$
\left\langle\omega_{p q}^{+}, \omega_{i j}^{-}\right\rangle=2 \pi i \sum_{x \in P^{1}} \operatorname{Res}_{x} \xi,
$$

which completes the proof.

By using forms

$$
\varphi_{j}=\frac{d t}{t-x_{j}}-\frac{d t}{t-x_{j+1}} \in \Gamma\left(\boldsymbol{P}^{1}, \Omega^{1}(\log D)\right), \quad 1 \leq j \leq n-1,
$$

we give bases for the spaces $\Gamma\left(\boldsymbol{P}^{1}, \Omega^{1}(\log D)\right) / \mathbf{C} \cdot \omega$ and $\Gamma\left(\boldsymbol{P}^{1}, \Omega^{1}(\log D)\right) / \mathbf{C}$ $\cdot(-\omega)$ by

$\varphi_{j}^{+} \in \Gamma\left(\boldsymbol{P}^{1}, \Omega^{1}(\log D)\right) / \mathbf{C} \cdot \omega, \quad \varphi_{j}^{-} \in \Gamma\left(\boldsymbol{P}^{1}, \Omega^{1}(\log D)\right) / \mathbf{C} \cdot(-\omega), \quad 1 \leq j \leq n-1$.

COROLlary. For the bases above, the intersection numbers are given as follows:

$$
\begin{gathered}
\left\langle\varphi_{j}^{+}, \varphi_{j}^{-}\right\rangle=2 \pi i\left(\frac{1}{\alpha_{j}}+\frac{1}{\alpha_{j+1}}\right), \\
\left\langle\varphi_{j}^{+}, \varphi_{j+1}^{-}\right\rangle=\left\langle\varphi_{j+1}^{+}, \overline{\varphi_{j}^{-}}\right\rangle=-\frac{2 \pi i}{\alpha_{j+1}}, \\
\left\langle\varphi_{j}^{+}, \varphi_{k}^{-}\right\rangle=0 \quad \text { if }|j-k| \geq 2 .
\end{gathered}
$$

\section{§3. Twisted Riemann's period relations}

In this section we assume $\alpha_{j} \notin \boldsymbol{Z}$. Let $\xi_{j}$ (resp. $\eta_{j}$ ) $1 \leq j \leq n-1$ be elements of $\Gamma\left(\Omega^{1}(\log D)\right)$ such that $\xi_{j}^{+}\left(\right.$resp $\left.\eta_{j}^{-}\right)$forms a basis of $\Gamma\left(\Omega^{1}(\log D)\right) / \mathbf{C} \cdot \omega$ (resp. $\left.\Gamma\left(\Omega^{1}(\log D)\right) / \mathbf{C} \cdot(-\omega)\right)$. Recall the de Rham expression:

$$
H_{c}^{1}\left(L^{\vee}\right) \simeq \frac{\operatorname{ker}\left\{\nabla^{\vee}: \Gamma_{c}\left(U, \mathscr{E}^{1}\right) \rightarrow \Gamma_{c}\left(U, \mathscr{E}^{2}\right)\right\}}{\nabla^{\vee} \Gamma_{c}\left(U, \mathscr{E}^{0}\right)} ;
$$

the natural inclusion

$$
\operatorname{ker}\left\{\nabla^{\vee}: \Gamma_{c}\left(U, \mathscr{E}^{1}\right) \rightarrow \Gamma_{c}\left(U, \mathscr{E}^{2}\right)\right\} \hookrightarrow \operatorname{ker}\left\{\Gamma\left(\mathscr{E}^{01}(-D)\right) \oplus \Gamma\left(\mathscr{E}^{10}\right) \rightarrow \Gamma\left(\mathscr{E}^{11}\right)\right\}
$$

induces the isomorphism (here the assumption $\alpha_{j} \notin \mathbf{N}-\{0\}$ is used)

$$
\left(H_{c}^{1}\left(L^{\vee}\right) \simeq\right) \frac{\operatorname{ker}\left\{\nabla^{\vee}: \Gamma_{c}\left(U, \mathscr{E}^{1}\right) \rightarrow \Gamma_{c}\left(U, \mathscr{E}^{2}\right)\right\}}{\nabla^{\vee} \Gamma_{c}\left(U, \mathscr{E}^{0}\right)}
$$




$$
\widetilde{\rightarrow} \frac{\operatorname{ker}\left\{\Gamma\left(\mathscr{E}^{01}(-D)\right) \oplus \Gamma\left(\mathscr{E}^{10}\right) \rightarrow \Gamma\left(\mathscr{E}^{11}\right)\right\}}{\nabla^{\vee} \Gamma\left(\mathscr{E}^{00}(-D)\right)}\left(\underset{\iota}{\stackrel{\tau}{\leftrightarrows}} \Gamma\left(\Omega^{1}(\log D)\right) / \mathbf{C} \cdot(-\omega)\right) .
$$

For each $\eta_{j}$ there exist (see $\left.\S 2(2)\right) \mu_{j} \in \Gamma\left(\mathscr{E}^{10}\right)$ and $h_{j} \in \Gamma\left(\mathscr{E}^{00}\right)$ such that

$$
\eta_{j}^{\vee}+\mu_{j}=\eta_{j}+\nabla^{\vee} h_{j} ;
$$

moreover by the isomorphism above there exist $f, \in \Gamma\left(\mathscr{E}^{00}(-D)\right)$ such that

$$
\eta_{j}^{\mathrm{c}}:=\eta_{j}^{\vee}+\mu_{j}+\nabla^{\vee} f_{j} \in \Gamma_{c}\left(U, \mathscr{g}^{1}\right),
$$

which form a basis of $\Gamma_{c}\left(U, \mathscr{E}^{1}\right)$. Let

$$
\gamma_{j}^{+} \in H_{1}\left(L^{\vee}\right), \quad \delta_{j}^{-} \in H_{1}(L)
$$

be bases of the twisted cycles. We use the following isomorphism called the Poincaré duality (without any condition):

$$
\theta_{c}: H_{1}\left(U, L^{\vee}\right) \simeq H^{1}\left(\Gamma_{c}\left(U, \mathscr{E}^{\vee}\right), \nabla^{\vee}\right) .
$$

Let us define the intersection matrices and the period matrices as follows:

$$
\begin{gathered}
I_{h}=\left(\begin{array}{ccc}
\left\langle\gamma_{1}^{+}, \delta_{1}^{-}\right\rangle & \cdots & \left\langle\gamma_{1}^{+}, \delta_{n-1}^{-}\right\rangle \\
\vdots & & \vdots \\
\left\langle\gamma_{n-1}^{+}, \delta_{1}^{-}\right\rangle & \cdots & \left\langle\gamma_{n-1}^{+}, \delta_{n-1}^{-}\right\rangle
\end{array}\right), I_{c h}=\left(\begin{array}{ccc}
\left\langle\xi_{1}^{+}, \eta_{1}^{-}\right\rangle & \cdots & \left\langle\xi_{1}^{+}, \eta_{n-1}^{-}\right\rangle \\
\vdots & & \vdots \\
\left\langle\xi_{n-1}^{+}, \eta_{1}^{-}\right\rangle & \cdots & \left\langle\xi_{n-1}^{+}, \eta_{n-1}^{-}\right\rangle
\end{array}\right) . \\
P^{+}=\left(\begin{array}{ccc}
\int_{r_{1}^{+}} \xi_{1}^{+} & \cdots & \int_{r_{n-1}^{+}} \xi_{1}^{+} \\
\vdots & & \vdots \\
\int_{r_{1}^{+}} \xi_{n-1}^{+} & \cdots & \int_{r_{n-1}^{+}} \xi_{n-1}^{+}
\end{array}\right), P^{-}=\left(\begin{array}{ccc}
\int_{\delta_{1}} \eta_{1}^{-} & \cdots & \int_{\delta_{n-1}^{-1}} \eta_{1}^{-} \\
\vdots & & \vdots \\
\int_{\delta_{1}^{-}}^{\eta_{n-1}^{-}} & \cdots & \int_{\delta_{n-1}^{-}}^{\eta_{n-1}^{-}}
\end{array}\right),
\end{gathered}
$$

where the intersection for twisted cycles are defined by

$$
\left\langle\gamma^{+}, \delta^{-}\right\rangle:=\int_{\delta^{-}} \theta_{c}\left(\gamma^{+}\right), \quad \gamma^{+} \in H_{1}\left(L^{\vee}\right), \quad \delta^{-} \in H_{1}(L) .
$$

Then we have the twisted Riemann's period relation:

THEOREM 2.

$$
P^{+t} I_{h}^{-1 t} P^{-}=I_{c h}, \quad \text { i.e. }{ }^{t} P^{-t} I_{c h}^{-1} P^{+}={ }^{t} I_{h}
$$

Proof. Let $\Theta=\left(\theta_{i j}\right)$ be the matrix expression of $\theta_{c}$ under the bases above: 


$$
\theta_{c}\left(\gamma_{j}^{+}\right)=\sum_{k} \theta_{k j} \eta_{k}^{c}
$$

The intersection numbers for twisted cycles are computed as follows:

$$
\begin{aligned}
\left\langle\gamma_{j}^{+}, \delta_{k}^{-}\right\rangle: & =\int_{\delta_{k}^{-}} \theta_{c}\left(\gamma_{j}^{+}\right)=\int_{\delta_{k}^{-}} \sum_{a} \theta_{a j} \eta_{a}^{\mathrm{c}} \\
& =\sum_{a} \theta_{a j} \int_{\delta_{k}^{-}} \eta_{a}+\nabla^{\vee} h_{a}+\nabla^{\vee} f_{a}=\sum_{a} \theta_{a j} \int_{\delta_{\bar{k}}^{-}} \eta_{a}^{-},
\end{aligned}
$$

that is

$$
I_{h}={ }^{\mathrm{t}} \Theta P^{-} .
$$

The $(k, j)$-components $\theta_{k j}$ of $\Theta$ are computed as follows:

$$
\begin{aligned}
\int_{\gamma_{j}^{+}} \xi_{a}^{+} & =\int \theta_{c}\left(\gamma_{j}^{+}\right) \wedge \xi_{a}^{+}=\int \sum_{k} \theta_{k j} \eta_{k}^{\mathrm{c}} \wedge \xi_{a} \\
& =\sum_{k} \theta_{k j} \int\left(\eta_{k}^{\vee}+\mu_{k}+\nabla^{\vee} f_{k}\right) \wedge \xi_{a} \\
& =\sum_{k} \theta_{k j} \int \eta_{k}^{\vee} \wedge \xi_{a}=\sum_{k}\left\langle\xi_{a}^{+}, \eta_{k}^{-}\right\rangle \theta_{k j},
\end{aligned}
$$

that is

$$
P^{+}=I_{c h} \Theta
$$

Eliminating $\Theta$ from the two equalities above, we get the relation.

\section{§4. Examples}

EXAmPle 1. Quadric relations for the Gauss hypergeometric functions.

For

$$
\begin{gathered}
n=3, x_{0}=x_{4}=\infty, x_{1}=0, x_{2}=1, x_{3}=1 / x(0<x<1), \\
\alpha_{1}=\alpha, \alpha_{2}=\gamma-\alpha, \alpha_{3}=-\beta, \alpha_{0}=\beta-\gamma,
\end{gathered}
$$

put

$$
\begin{aligned}
u & =t^{\alpha}(1-t)^{\gamma-\alpha}(1-x t)^{-\beta}, \\
\varphi_{1}=\left(\frac{d t}{t-x_{1}}-\frac{d t}{t-x_{2}}\right) & =\frac{d t}{t(1-t)}, \varphi_{3}=\left(\frac{d t}{t-x_{3}}-\frac{d t}{t-x_{4}}\right)=\frac{-x d t}{1-x t},
\end{aligned}
$$

$\gamma_{1}^{+}, \gamma_{3}^{+} \in H_{1}\left(U, L^{\vee}\right)$ and $\gamma_{1}^{-}, \gamma_{3}^{-} \in H_{1}(U, L)$, (see Figure), then we have 


$$
\begin{gathered}
P^{+}=\left(\begin{array}{cc}
\int_{0}^{1} u \varphi_{1} & \int_{1 / x}^{\infty} u \varphi_{1} \\
\int_{0}^{1} u \varphi_{3} & \int_{1 / x}^{\infty} u \varphi_{3}
\end{array}\right), P^{-}=\left(\begin{array}{cc}
\int_{0}^{1} u^{-1} \varphi_{1} & \int_{1 / x}^{\infty} u^{-1} \varphi_{1} \\
\int_{0}^{1} u^{-1} \varphi_{3} & \int_{1 / x}^{\infty} u^{-1} \varphi_{3}
\end{array}\right), \\
I_{h}=-\left(\begin{array}{cc}
d_{12} / d_{1} d_{2} & 0 \\
0 & d_{30} / d_{3} d_{0}
\end{array}\right), I_{c h}=2 \pi i\left(\begin{array}{cc}
1 / \alpha+1 /(\gamma-\alpha) & 0 \\
0 & -1 / \beta+1 /(\beta-\gamma)
\end{array}\right) .
\end{gathered}
$$

By the help of the well-known formulae

$$
\begin{gathered}
\int_{0}^{1} u \varphi_{1}=B(\alpha, \gamma-\alpha) F(\alpha, \beta, \gamma ; x), \\
\int_{1 / x}^{\infty} u \varphi_{1}=-(-1)^{\gamma-\alpha-\beta} x^{1-\gamma} B(\beta-\gamma+1,-\beta+1) \\
\times F(\beta-\gamma+1, \alpha-\gamma+1,2-\gamma ; x),
\end{gathered}
$$

the identity

$$
P^{+t} I_{h}^{-1 t} P^{-}=I_{c h},
$$

leads quadratic identities for hypergeometric functions in [SY]: the $(1,2)-$ component yields the formula presented in Introduction

$$
\begin{aligned}
& F(\alpha, \beta, \gamma ; x) F(1-\alpha, 1-\beta, 2-\gamma ; x) \\
& =F(\alpha+1-\gamma, \beta+1-\gamma, 2-\gamma ; x) F(\gamma-\alpha, \gamma-\beta, \gamma ; x),
\end{aligned}
$$

and the $(1,1)$-component yields

$$
\begin{gathered}
F(\alpha, \beta, \gamma ; x) F(-a,-\beta,-\gamma ; x)-1 \\
=\frac{\alpha \beta(\gamma-\alpha)(\gamma-\beta)}{\gamma^{2}(\gamma+1)(\gamma-1)} F(\beta-\gamma+1, \alpha-\gamma+1,-\gamma+2 ; x) \\
\quad \times F(\gamma-\beta+1, \gamma-\alpha+1, \gamma+2 ; x) .
\end{gathered}
$$

EXAMPLE 2. Quadric relations for Lauricella's hypergeometric function. Lauricella's hypergeometric function $F_{D}$ of $m$-variable is defined by

$$
F_{D}(\alpha, \beta, \gamma ; z)=\sum_{n_{1}, n_{2}, \ldots, n_{m}=0}^{\infty} \frac{(\alpha)_{n_{1}+\cdots+n_{m}}\left(\beta_{1}\right)_{n_{1}} \cdots\left(\beta_{m}\right)_{n_{m}}}{(\gamma)_{n_{1}+\cdots+n_{m}}(1)_{n_{1}} \cdots(1)_{n_{m}}} z_{1}^{n_{1}} \cdots z_{m}^{n_{m}},
$$

where

$$
z=\left(z_{1}, \ldots, z_{m}\right), \quad \beta=\left(\beta_{1}, \ldots, \beta_{m}\right) ;
$$

the series admits the integral representation 


$$
F_{D}(\alpha, \beta, \gamma ; z)=\frac{\Gamma(\gamma)}{\Gamma(\alpha) \Gamma(\gamma-\alpha)} \int_{0}^{1} t^{\alpha-1}(1-t)^{\gamma-\alpha-1}\left(1-z_{1} t\right)^{-\beta_{1}} \cdots\left(1-z_{m} t\right)^{-\beta_{m}} d t
$$

Put

$$
\begin{gathered}
n=m+2, x_{0}=\infty, x_{1}=0, x_{2}=1, x_{j+2}=1 / z_{j}(1 \leq j \leq m), \\
\alpha_{0}=\alpha_{m+3}=\beta_{1}+\cdots+\beta_{m}-\gamma, \alpha_{1}=\alpha, \alpha_{2}=\gamma-\alpha, \alpha_{j+2}=-\beta_{j}(1 \leq j \leq m), \\
u=t^{\alpha}(1-t)^{\gamma-\alpha}\left(1-z_{1} t\right)^{-\beta_{1}} \cdots\left(1-z_{m} t\right)^{-\beta_{m}}, \\
\xi_{j}=\left(\frac{1}{t-x_{1}}-\frac{1}{t-x_{j+1}}\right) d t, \eta_{j}=\left(\frac{1}{t-x_{j+1}}-\frac{1}{t-x_{0}}\right) d t(1 \leq j \leq m+1), \\
\gamma_{j}^{+}, H_{1}\left(U, L^{\vee}\right), \gamma_{j}^{-} \in H_{1}(U, L)(1 \leq j \leq m), \text { (see Figure). }
\end{gathered}
$$

The (1,1)-component of

$$
{ }^{\mathrm{t}} P^{-} I_{c h}^{-1} P^{+}={ }^{t} I_{h}
$$

reads

$$
\left(\int_{0}^{1} u^{-1} \eta_{1}, \ldots, \int_{0}^{1} u^{-1} \eta_{m+1}\right) I_{c h}^{-1}\left(\int_{0}^{1} u \xi_{1}, \ldots, \int_{0}^{1} u \xi_{m+1}\right)=I_{h}(1,1) .
$$

Since the (1,1)-component of $I_{h}$ is $-\left(e^{2 \pi i \gamma}-1\right) /\left(\left(e^{2 \pi i \alpha}-1\right)\left(e^{2 \pi i(\gamma-\alpha)}-1\right)\right)$, and

$$
I_{c h}^{-1}=-\frac{1}{2 \pi i}\left(\begin{array}{ccccc}
\alpha-\gamma & 0 & 0 & \cdots & 0 \\
0 & \beta_{1} z_{1} & 0 & \cdots & 0 \\
0 & 0 & \beta_{2} z_{2} & \cdots & 0 \\
\vdots & \vdots & \cdots & \ddots & \vdots \\
0 & 0 & \cdots & 0 & \beta_{m} z_{m}
\end{array}\right)
$$

we have the following formula:

$$
\begin{gathered}
F_{D}(\alpha, \beta, \gamma ; z) F_{D}(1-\alpha,-\beta,-\gamma+1 ; z)-1 \\
=\frac{\gamma-\alpha}{\gamma(\gamma-1)} \sum_{j=1}^{m} \beta_{j} z_{j} F_{D}\left(\alpha, \beta+e_{j}, \gamma+1 ; z\right) F_{D}\left(-\alpha+1,-\beta+e_{j},-\gamma+2 ; z\right),
\end{gathered}
$$

where

$$
e_{j}=(\ldots, 0,1,0, \ldots)
$$

Remark. Once the inversion formula for the beta function is obtained as an example of the twisted Riemann's period relations, the inversion formula for the gamma function can be obtained as a special case of beta's as follows: 


$$
\begin{aligned}
\Gamma(\alpha) \Gamma(-\alpha)=B(\alpha, & -\alpha / 2) B(-\alpha, \alpha / 2) \\
& =\frac{-2 \pi i}{\alpha} \frac{\exp (\pi i \alpha)}{\exp (2 \pi i \alpha)-1}=-\frac{1}{\alpha} \frac{\pi}{\sin \pi \alpha}
\end{aligned}
$$

namely $\Gamma(a) \Gamma(1-\alpha)=\pi / \sin \pi \alpha$. Since the gamma function can be thought of a confluent beta function (see the integral representations of these functions in the beginning of Introduction), this formula suggests a confluent version of our intersection theory.

\section{REFERENCES}

[Aom1] K. Aomoto, On vanishing of cohomology attached to certain many valued meromorphic functions, J. Math. Soc. Japan, 27 (1975), 248-255.

[Aom2] - On the structure of intergrals of power product of linear functions, Sci. Papers College of General Ed, Univ. of Tokyo, 27 (1977), 49-61.

[Cho1] K. Cho, Intersection theory for twisted cohomologies and twisted Riemann's period relations II-On Riemann srufaces, preprint.

[Cho2] - Intersectin theory for twisted cohomologies and twisted Riemann's period relations III-On $P^{n}$, preprint.

[CY] K. Cho and M. Yoshida, Comparison of (co)homologies of branched covering spaces and twisted ones of basespaces I, Kyushu J. Math., 48 (1994), 111-122.

[Del1] P. Deligne, Equations différentielles à points singuliers réguliers, Lect. Notes in Math., 163, Springer, 1970.

[De12] - Théorie de Hodge II, Publ. Math., Inst. Hautes Etud. Sci., 40 (1972), $5-57$.

[EV1] H. Esnault and E. Viehweg, Logarithmic De Rham complexes and vanishing theorems, Invent. Math., 86 (1986), 161-194.

[EV2] - Lectures on Vanishing Theorems, Birkhäuser, 1992.

[ESV] H. Esnault, V. Schechtman and E. Viehweg, Cohomology of local systems on the complement of hyperplanes, Invent, Math., 109 (1992), 557-561.

[For] O. Forster, Lectures on Riemann Surfaces, GTM 81, Springer, 1977.

[GH] P. Griffiths and J. Harirs, Principles of Algebraic Geometry, John Wiley \& Sons, Inc., 1978.

[IK1] K. Iwasaki and M. Kita, Exterior power structure of the twisted de Rham cohomology of the complement of real Veromese arrangements, to appear in J. Math. Pures et Appl.

[IK2] - Twisted homology of the configuration space of $n$-points with application to hypergeometric functions, preprint UTMS 94-11, (1944).

[IKSY] K. Iwasaki H. Kimura, S. Shimomura and M. Yoshida, From Gauss to Painlevé, Vieweg, 1991.

[Kit] M. Kita, On the hypergeometric functions in several variables II-On the Wronskian of the hypergeometric functions of type $(n+1, m+1)-$, J. Math. Soci. Japan, 45 (1993), 645-669.

[KM] M. Kita and K. Matsumoto, Duality for hypergeometric furctions and inrariant Gauss-Manin systems preprint. 
[KN] M. Kita and M. Noumi, On the structure of cohomology groups attached to integrals of certain many valued analytic functions, Japan. J. Math., 9 (1983), $113-157$.

[KY1, 2] M. Kita and M. Yoshida, Intersection theory for twisted cycles I, II, Math. Nachrichten, 166 (1994), 287-304, 168 (1994), 171-190.

[SY] T. Sasaki and M. Yoshida, Tensor Products of Linear Differential Equations II - New formulae for the hypergeometric functions -, Funkcialaj Ekvacioj, 33 (1990), 527-549.

[Yos] M. Yoshida, Fuchsian Differential Equations, Vieweg, 1987.

K. Cho

Graduate School of Mathematics

Kyushu University

Hakozaki, Higashi-ku

Fukuoka 812, Japan

K. Matsumoto

Department of Mathematics

Faculty of Science

Hiroshima University

Kagamiyama, Higashi-Hiroshima 739, Japan 